\title{
Involvement of Non-Coding RNAs in Chemo- and Radioresistance of Nasopharyngeal Carcinoma
}

Jiaxin Xiao

Xiusheng $\mathrm{He}$

Hunan Province Key Laboratory of Tumour Cellular \& Molecular Pathology Cancer Research Institute, Hengyang Medical College of University of South China, Hengyang, 42100I, Hunan Province, People's Republic of China
Correspondence: Xiusheng He Hunan Province Key Laboratory of Tumour Cellular \& Molecular Pathology Cancer Research Institute, Hengyang Medical College of University of South China, Hengyang, 42I00I, Hunan Province, People's Republic of China Email hexiusheng168@I63.com

\begin{abstract}
The crucial treatment for nasopharyngeal carcinoma (NPC) is radiation therapy supplemented by chemotherapy. However, long-term radiation therapy can cause some genetic and proteomic changes to produce radiation resistance, leading to tumour recurrence and poor prognosis. Therefore, the search for new markers that can overcome the resistance of tumor cells to drugs and radiotherapy and improve the sensitivity of tumor cells to drugs and radiotherapy is one of the most important goals of pharmacogenomics and cancer research, which is important for predicting treatment response and prognosis. Non-coding RNAs (ncRNAs), such as microRNAs (miRNAs) and long non-coding RNAs (lncRNAs), may play important roles in regulating chemo- and radiation resistance in nasopharyngeal carcinoma by controlling the cell cycle, proliferation, apoptosis, and DNA damage repair, as well as other signalling pathways. Recent research has suggested that selective modulation of ncRNA activity can improve the response to chemotherapy and radiotherapy, providing an innovative antitumour approach based on ncRNA-related gene therapy. Therefore, ncRNAs can serve as biomarkers for tumour prediction and prognosis, play a role in overcoming drug resistance and radiation resistance in NPC, and can also serve as targets for developing new therapeutic strategies. In this review, we discuss the involvement of ncRNAs in chemotherapy and radiation resistance in NPC. The effects of these molecules on predicting therapeutic cancer are highlighted.
\end{abstract}

Keywords: miRNAs, lncRNAs, nasopharyngeal carcinoma, chemo- and radioresistance

\section{Introduction}

Nasopharyngeal carcinoma (NPC) is an epithelial carcinoma that most commonly affects the inner mucosa of the nasopharynx. It is a common malignant tumour of the head and neck, mostly found in the pharyngeal crypt, with a high incidence in Southeast Asia and Southern China, and the non-keratinized subtype associated with EB virus (EBV) infection constitutes the majority of cases $(>95 \%)$ in endemic areas. ${ }^{1}$ Due to its unique anatomical location and high sensitivity to radiotherapy, the main treatment for patients with early and locally advanced nasopharyngeal carcinoma is radiation therapy. ${ }^{1,2}$ Multidrug chemotherapy with platinum is the basic treatment option for patients with recurrent nasopharyngeal carcinoma. ${ }^{3}$ One of the main problems in the treatment of patients with nasopharyngeal carcinoma is the resistance of cancer cells to radiotherapy and chemotherapy, which tends to lead to unsatisfactory treatment results, tumour recurrence and poor prognosis., ${ }^{2,45}$ Hence, the main objective of current clinical research is to implement appropriate strategies through which patients can overcome resistance during chemotherapy or radiotherapy. 
Understanding the molecular mechanisms that lead to treatment resistance and identifying new targets to improve treatment efficiency may help oncologists develop personalized directions for cancer treatment. ${ }^{6,7}$ Discovering and validating new predictive and prognostic biomarkers may serve as a vital tool to provide better treatment outcomes and directions for patients requiring specific treatment regimens, providing a better approach for tailoring therapy by providing more appropriate treatment options based on particular patients. ${ }^{8,9}$ Epstein-Barr virus (EBV)-encoded products, microRNAs (miRNAs), long non-coding RNAs (lncRNAs) and circular RNAs (circRNAs) have been identified in existing research as having common signalling pathways that perform many functions in NPC. ${ }^{10}$ Non-coding RNAs, including miRNAs and lncRNAs, have powerful biological functions in cancer. The involvement of ncRNAs in the regulation of tumour proliferation, migration and invasion has been demonstrated in many studies ${ }^{1-13}$ and is related to chemotherapy resistance and radiation resistance. ${ }^{14,15}$

In this review, the involvement of miRNAs and lncRNAs in chemotherapy and radiotherapy for nasopharyngeal carcinoma will be described, and a preliminary investigation of the molecular mechanisms that may be involved is conducted.

\section{Non-coding RNAs Involved in Radioresistance of NPC}

After radiation treatment of tumour cells, the DNA strand is disrupted, and the cells die, losing the ability to proliferate indefinitely. However, the increase in tumour size, decrease in oxygen and dysregulation of various genes can lead to radiation tolerance of tumour cells, resulting in radioresistance and reduced sensitivity to treatment. ${ }^{16}$ In radiation resistance, the gene-related causes mainly include the decrease of apoptotic gene expression, the overexpression of proliferation and antiapoptotic genes, the intensification of expression of genes that mediate DNA damage and repair, or the neglect of the expression of cell cycle regulation genes. The most specific reason for this is the decrease in apoptotic cells after radiation exposure. ${ }^{17-20}$ ncRNAs have been shown to be aberrantly expressed in a large number of cancers, suggesting a potential role in cancer pathogenesis. miRNAs and lncRNAs, in particular, play an important role in the regulation of drug resistance in NPC by managing the proliferation cell cycle, DNA damage repair, and apoptosis or other key cellular signalling pathways. ${ }^{21-24}$

\section{MiRNAs Affect the Sensitivity of NPC to Radiotherapy}

MicroRNAs (miRNAs), which are approximately 22 nucleotides in length, are small non-coding RNA molecules that play an important role in post-transcriptional regulation and thus influence the development of a variety of diseases. Several studies have shown that miRNAs are dysregulated at the expression level in various cancers and that dysregulated miRNAs in turn affect cell proliferation, cell death inhibition, metastasis and angiogenesis in different cancers, which may be related to multiple mechanisms. ${ }^{25}$ Several miRNAs have been shown to regulate radiosensitivity in nasopharyngeal carcinoma.

The experimental results of Zhang et al demonstrated that miR-451 overexpression enhanced the radiosensitivity of NPC cells by a mechanism associated with inhibition of the repair of irradiation-induced double-strand breaks (DSBs) and increased apoptosis. Additionally, low expression of RAB14 restored ionizing radiation (IR)-induced miR-451-mediated DSBs. miR-451 directly targets RAB14 to enhance the radiosensitivity of NPC cells, and thus, miR-451 may be a potential marker of radiosensitization. In addition, miR-451 is downregulated in several tumours, including glioma, lung cancer, oesophageal cancer and breast cancer. ${ }^{26}$ Wang et al recognized that miR206 was expressed at low levels in radiation-resistant NPC cells. Furthermore, restoration of miR-206 in CNE2-IR cells increased the radiosensitivity of NPC cells, and inhibition of miR-206 in CNE2 cells decreased the radiosensitivity. The authors concluded that miR-206 sensitizes NPC cells to irradiation by targeting IGF1. ${ }^{27}$ Interestingly, miR-206 expression was reduced in a variety of cancers, including breast and lung cancers, and reversed cisplatin resistance in lung adenocarcinoma cells. ${ }^{28,29}$ This suggests that in the search for key therapeutic targets in nasopharyngeal carcinoma, we can gain inspiration from key molecules that have been identified in other cancers.

Tian et al demonstrated that JAMA in NPC can be directly targeted by miR-124; thus, stem cell properties are suppressed, and the radiosensitivity of nasopharyngeal carcinoma is improved. ${ }^{30}$ It has been reported that COL1A1 induces radioresistance, and in nasopharyngeal carcinoma, 
miR-29a targets COL1A1, cell proliferation capacity is inhibited and apoptosis is enhanced after irradiation, reversing COL1A1 radioresistance to some extent; thus, miR-29a can be used as a biomarker for radiosensitization. ${ }^{31}$ In addition, a number of studies have demonstrated that a variety of miRNAs play radiosensitization roles in nasopharyngeal carcinoma (Table 1). However, radiation resistance is promoted by other miRNAs. A study showed that miR-193a-3p directly targets the SRSF2 gene, resulting in increased radioresistance in nasopharyngeal carcinoma. ${ }^{32}$

Huang et al demonstrated that miR-150 expression was upregulated and GSK3 $\beta$ protein expression was downregulated in nasopharyngeal carcinoma CNE-2R cells. miR-150 expression was inhibited, and the radioresistance of CNE-2R cells was reversed, whereas the radioresistance of CNE-2 cells was enhanced after overexpression of miR-150. miR-150b was shown to increase the radioresistance of CNE- 2 cells by directly targeting GSK3 $\beta$ to elicit these responses. ${ }^{33}$ It has also been shown that miR-182-5p upgrades radioresistance in nasopharyngeal carcinoma by directing the expression of BNIP3. ${ }^{34}$ Therefore, miRNAs may be a potential target for reversing radioresistance in NPC cells. Therefore, in future studies on nasopharyngeal carcinoma, we can improve the radiosensitivity and reverse radioresistance of nasopharyngeal carcinoma cells by regulating the expression level of miRNA. miRNAs can be used as biomarkers to provide new ideas for the treatment of nasopharyngeal carcinoma.

\section{Mechanisms of miRNAs in Radiotherapy Resistance in Nasopharyngeal Carcinoma}

Previous work has shown that miRNAs can affect tumour radioresistance by interfering with multiple pathways, ${ }^{35}$ including sensing DNA damage, ${ }^{36}$ repairing DNA doublestrand breaks (DSBs), ${ }^{37}$ and activating the cell cycle

Table I ncRNAs Affect the Radiosensitivity of miRNAs in NPC

\begin{tabular}{|c|c|c|c|c|}
\hline ncRNAs & Expression & Targets & Functional Regulation & References \\
\hline miR-45I & $\uparrow$ & $\mathrm{RAB} \mid 4$ & $\uparrow$ Radio-sensitivity & {$[26]$} \\
\hline miR-206 & $\uparrow$ & IGFI & $\uparrow$ Radio-sensitivity & {$[27]$} \\
\hline miR-593 & $\uparrow$ & MDRI & $\uparrow$ Radio-sensitivity & [109] \\
\hline miR-I 24 & $\uparrow$ & PDCD6 & $\uparrow$ Radio-sensitivity & [30] \\
\hline miR- 24 & $\uparrow$ & SPI & $\uparrow$ Radio-sensitivity & {$[110]$} \\
\hline miR-I24 & $\uparrow$ & JAMA & $\uparrow$ Radio-sensitivity & {$[\mathrm{III}]$} \\
\hline miR-29-3p & $\uparrow$ & COLIAI 3'-UTR & $\uparrow$ Radio-sensitivity & {$[31]$} \\
\hline $\operatorname{miR}-24-3 p$ & $\uparrow$ & Jabl/CSN5 & $\uparrow$ Radio-sensitivity & {$[112]$} \\
\hline miR-9-5p & $\uparrow$ & HK2 & $\uparrow$ Radio-sensitivity & {$[113]$} \\
\hline miR-2I & $\downarrow$ & $*$ & $\uparrow$ Radio-sensitivity & {$[114]$} \\
\hline$m i R-483-5 p$ & $\uparrow$ & DAPKI & $\downarrow$ Radio-sensitivity & [115] \\
\hline miR- BART8-3p & $\uparrow$ & ATM/ATR & $\downarrow$ Radio-sensitivity & {$[116]$} \\
\hline miR-383-5p & $\uparrow$ & RBM3 & $\uparrow$ Radio-sensitivity & {$[117]$} \\
\hline miR-139-5p & $\uparrow$ & EMT & $\uparrow$ Radio-sensitivity & [75] \\
\hline $\operatorname{miR}-222$ & $\uparrow$ & PTEN & $\downarrow$ Radio-sensitivity & [118] \\
\hline miR-18Ia & $\uparrow$ & RKIP & $\downarrow$ Radio-sensitivity & [119] \\
\hline miR-193a-3p & $\uparrow$ & SRSF2 & $\downarrow$ Radio-sensitivity & [32] \\
\hline $\operatorname{miR}-205$ & $\uparrow$ & PTEN & $\downarrow$ Radio-sensitivity & [43] \\
\hline miR-203 & $\uparrow$ & IL8/AKT & $\uparrow$ Radio-sensitivity & {$[120]$} \\
\hline miR-182-5p & $\uparrow$ & BNIP3 & $\downarrow$ Radio-sensitivity & [34] \\
\hline miR-372 & $\uparrow$ & p53 & $\uparrow$ Radio-sensitivity & {$[121]$} \\
\hline $\operatorname{miR}-23 a$ & $\downarrow$ & IL-8/Stat3 & $\downarrow$ Radio-sensitivity & {$[122]$} \\
\hline miR-I50 & $\uparrow$ & Glycogen synthase kinase- $3 \beta$ & $\downarrow$ Radio-sensitivity & [33] \\
\hline Lnc ANRIL & $\downarrow$ & $\mathrm{miR}-125 \mathrm{a}$ & $\uparrow$ Radio-sensitivity & [24] \\
\hline Lnc XIST & $\downarrow$ & $\operatorname{miR}-29 c$ & $\uparrow$ Radio-sensitivity & [123] \\
\hline Lnc ANCR & $\uparrow$ & PTEN & $\downarrow$ Radio-sensitivity & [59] \\
\hline Lnc MINCR & $\uparrow$ & miR-223/ZEB I & $\downarrow$ Radio-sensitivity & {$[60]$} \\
\hline Lnc PTPRG-ASI & $\uparrow$ & miR-194-3p & $\downarrow$ Radio-sensitivity & [124] \\
\hline Lnc PVTI & $\uparrow$ & $*$ & $\downarrow$ Radio-sensitivity & [61] \\
\hline Lnc MALATI & $\downarrow$ & $\mathrm{miR}-\mathrm{I} / \mathrm{slug}$ axis & $\uparrow$ Radio-sensitivity & {$[125]$} \\
\hline
\end{tabular}

Notes: $\uparrow$ Up-regulated and $\downarrow$ down-regulated ncRNAs in NPC, “*” target not specified. 
checkpoint, $^{38}$ apoptosis, ${ }^{39}$ and autophagy. ${ }^{40}$ Apoptosis, regulation of DNA damage repair, and regulation of the cell cycle are briefly described below.

Aberrant expression of apoptosis-associated genes can suppress ionizing radiation-induced apoptosis of tumour cells and increase their survival rate, thus promoting NPC radiation resistance. ${ }^{16} \mathrm{Bcl}-2$ is a gene that inhibits apoptosis. At least 19 congeners regulate the mitochondriadependent pathway of apoptosis and control the release of cytochrome $\mathrm{c}$ and other cytokines. ${ }^{41,42}$ Huang et al showed that the NF- $\kappa \mathrm{B}$, Wnt and P53 signalling pathways are associated with radioresistance and can also regulate apoptosis and the cell cycle. miR-19b-3p may act through these pathways. The apoptosis and cyclic effects of miR$19 b-3 p$ were evaluated by flow cytometry. The authors found that the apoptotic percentages of CNE1 and CNE2 cells decreased significantly after miR-19b-3p overexpression. The apoptotic percentages of CNE1 and CNE2 cells with lower miR-19b-3p expression were significantly higher. ${ }^{18}$ In addition, miR-125b, miR-205 and miR-21 reduce radiosensitivity in nasopharyngeal carcinoma by targeting Bcl-2 gene family proteins. ${ }^{43-45}$

ATM encodes a protein involved in the cell cycle and DNA damage repair. This protein belongs to the PI3/PI4 kinase family and is an important cell cycle checkpoint kinase that regulates the tumour suppressor proteins p53 and BRCA1, the checkpoint kinase CHK2, the checkpoint proteins RAD17 and RAD9, the DNA repair protein NBS1, and many other downstream proteins. $^{23,46}$ Endogenous ATM is the target of EBV-encoded miRNAs (Bart 5-5P, BART7-3p, BART9-3p and BART14-3p). ${ }^{22,47}$ When cells are irradiated with nonlethal doses and singleor double-stranded DNA breaks are repaired, cells can survive without apoptosis. This is one of the causes of radiation resistance. JAB1 (c-Jun activation domain binding protein-1) plays an important role in the repair of DNA double-strand breaks; DNA breaks spontaneously due to its deletion and histone $\gamma-\mathrm{H} 2 \mathrm{AX}$ expression increases in response, so JAB1 maintains genomic stability. ${ }^{21,48,49}$

Cell cycle distribution directly affects the radiosensitivity of nasopharyngeal carcinoma (NPC) cells, and thus miRNAs also mediate radiotherapy resistance in NPC through regulation of the cell cycle. Because cells differ in their sensitivity to radiation at different stages, cell cycle proteins, cell cycle protein-dependent cell kinase (CDK) and CDK kinase inhibitors (CDKIs) comprise the cell cycle regulatory system in the available studies. Previous studies have shown that overexpression of miR-
188 inhibits cell proliferation, tumour colony formation and $\mathrm{G} 1 / \mathrm{S}$ cell cycle transition in human nasopharyngeal carcinoma CNE cells. E2F transcription is downregulated by inhibiting $\mathrm{Rb}$ phosphorylation, thereby inhibiting CDK4 and CDK2 mRNA and protein expression, and thus enhancing the radiosensitivity of nasopharyngeal carcinoma cells. ${ }^{50} \mathrm{Lu}$ et al showed that miR-26a expression was reduced in nasopharyngeal carcinoma tissues and cells. miR-26a targets the EZH2 oncogene and reduces the expression level of EZH2 to inhibit cell growth and cell cycle progression. miR-26a inhibits the expression levels of C-myc, cell cycle proteins D3 and E2, and cell cycle protein-dependent kinases CDK4 and CDK6 while enhancing the expression of the CDK inhibitors p14ARF and p21CIP1 in an EZH2-dependent manner, thereby inducing cell cycle arrest in the G1 phase and acting as a radiosensitizer. ${ }^{51}$ It has also been found that miR-663 promotes the proliferation and cell cycle progression of nasopharyngeal carcinoma cells by directly targeting CDKN2A, suggesting that miR-663 may be a potential therapeutic target for the treatment of nasopharyngeal carcinoma. $^{52}$

\section{LncRNAs Affect the Sensitivity of Radiotherapy for NPC}

Long noncoding RNAs (lncRNAs) play a key regulatory role in genomic blotting, gene expression, cellular transfer, etc. They are transcripts longer than 200 nucleotides. ${ }^{53}$ There is growing evidence that IncRNAs can promote tumour malignancy in many tumours, including nasopharyngeal carcinoma, in addition to exerting an inhibitory effect on tumour growth and metastasis. ${ }^{54-57}$ In addition, some studies have confirmed the role of lncRNAs in radiotherapy of nasopharyngeal carcinoma. The research results of $\mathrm{Hu}$ et al proved that after low expression of lncRNA ANRIL negatively regulated the expression of miR-125a, the proliferative capacity of NPC cells was reduced, apoptosis was increased, and the sensitivity of NPC to radiation was elevated. ${ }^{24}$ Han et al found that the expression of XIST and miR-29c varied inversely in response to irradiation. Mechanistically, the authors validated the direct binding site of miR-29c on XIST. Rescue experiments further revealed that miR-29c inhibition abolished XIST knockdown-induced cell proliferation suppression and radiosensitivity increase in NPC cells, suggesting that XIST knockdown inhibited proliferation and improved radiosensitivity of NPC cells by upregulating miR-29c. ${ }^{58}$ Ma et al 
demonstrated that lnc ANCR can mediate the functional regulation of the EZH2 and PTEN promoters, thus inhibiting PTEN expression, promoting the proliferative capacity of NPC cells and inducing radioresistance. Thus, it can be hypothesized that low expression of lnc ANCR in nasopharyngeal carcinoma can inhibit radioresistance and play a sensitizing role. ${ }^{59}$ The experiments of Zhong et al illustrate that the lncRNA MINCR acts as a ceRNA for miR223 to positively regulate ZEB1. After low expression of MINCR, the PI3K/AKT axis associated with autophagy is inactivated, and miR-223 can target ZEB1, resulting in enhanced radiosensitivity of NPC. Therefore, we can provide new targets and new perspectives for the effective treatment of NPC by reducing the expression level of miRNAs that promote radioresistance. ${ }^{60} \mathrm{He}$ et al proved that PVT1 knockdown in NPC cells decreased ATM/Chk2/ p53 activation phosphorylation, weakened DNA repair ability, increased tumour apoptosis, and enhanced radiosensitivity. ${ }^{61}$ Therefore, lncRNAs can be used as potential biomarkers and therapeutic targets for nasopharyngeal carcinoma, and we can obtain inspiration from lncRNAs to obtain new ideas for reversing the radiation resistance of nasopharyngeal carcinoma.

\section{Mechanisms of IncRNAs in Radiotherapy Resistance in Nasopharyngeal Carcinoma}

In current studies, lncRNAs regulate the radiosensitivity of nasopharyngeal carcinoma mainly by mediating DNA damage, regulating the cell cycle, and targeting miRNAs. The expression of lncRNA-PVT1 was elevated in nasopharyngeal carcinoma patients, and low expression of the PVT1 gene promoted the radiosensitivity of nasopharyngeal carcinoma cells in vitro and in vivo, which may be associated with an increased apoptosis rate after IR. In addition, low expression of the PVT1 gene could decrease the phosphorylation levels of ATM, p53 and CHK2, key mediators of the DNA damage response. ${ }^{61}$ This suggests that PVT1 knockdown inhibited the repair ability of DSBs. HIF- $1 \alpha$ is a key factor in the response to hypoxic stress, and Wang et al further showed that PVT1 increased the stability of HIF-1 $\alpha$ in NPC cells. ${ }^{62}$ In addition, lncRNAs can also target cyclins to influence radiosensitivity. Wang et al found that IR-induced and Cur-reversed differentially expressed lncRNA AK294004 negatively regulates cell cycle protein D1 (CCND1), suggesting that lnc AK294004 can directly target CCND1. This study demonstrates that Cur enhances the radiosensitivity of NPC cells and that the function of radiation-resistant lncRNAs is reversed, suggesting an important role of lncRNAs in IRinduced radiation resistance. ${ }^{63}$ In addition, lncRNAs can modulate the radiosensitivity of nasopharyngeal carcinoma by targeting miRNAs or Mir-mediated signalling axes. It has been shown that IncRNAs and miRNAs can interact with each other in cancer progression. ${ }^{64,65}$ Han et al proved that lnc $\mathrm{C} 00114$ contributed to the progression and radioresistance of NPC by activating the ERK/JNK signalling pathway by targeting miR-203. Our study provided a novel theoretical basis for NPC treatment and antagonism to radioresistance. ${ }^{66}$ Huang et al proved that lncRNA FAM133B-2 represses the radioresistance of nasopharyngeal cancer cells by targeting the miR-34a-5p/ CDK6 axis. $^{67} \mathrm{Lu}$ et al demonstrated that lnc NEAT1 increased radioresistance and induced an EMT phenotype by eliminating the inhibitory effect of miR-204 on ZEB1 ${ }^{68}$ Understanding the mechanism of lncRNA radiation sensitization and reverse radiation resistance in nasopharyngeal carcinoma can provide a new direction for the treatment of nasopharyngeal carcinoma, which can be further explored in future studies.

\section{Non-coding RNAs Involved in Drug Resistance of NPC}

Nasopharyngeal carcinoma has a relatively high sensitivity to radiation; therefore, radiation therapy (RT) has been used as the first treatment option in the previous clinical management of NPC. However, NPC is not diagnosed until it has progressed to a locally advanced stage. The outcome and prognosis of radiation therapy alone are poor, with a low five-year survival rate. Therefore, the exploration of chemotherapy (CT) has deepened. Current studies have shown that NPC is sensitive not only to radiation but also to chemotherapy, and the use of various chemotherapeutic agents is gradually gaining ground. ${ }^{69}$ Therefore, advanced nasopharyngeal carcinoma is usually treated with a combination of radiotherapy and chemotherapy (CRT). Currently, CT is usually combined with RT in most patients with non-metastatic stage III/IV nasopharyngeal carcinoma. Especially since the publication of the results of the 009919 intergroup study, concurrent chemotherapy-radiotherapy (CRT) and adjuvant CT have been widely accepted as the standard of care for the treatment of patients with stage III and IV NPC. For those with recurrent NPC, the standard of care is multidrug chemotherapy with platinum-based agents. However, chemoresistance is a major obstacle to curing patients with 
recurrent NPC. Therefore, it is important to clarify the mechanism of chemoresistance in NPC. Modern considerations have shown that ncRNAs, particularly miRNAs and IncRNAs, may play an imperative role in NPC resistance by mediating efflux, death escape, upgrading DNA repair, EMT, CSC, EBV, exosomes, and apoptosis. ${ }^{5}$ In addition, ncRNAs can modulate the function of targets of drug action and regulate genes related to drug metabolism and transport. ${ }^{70}$ In addition, inactivation of oncogenic miRNAs promoted the expression of target tumour suppressor genes, while activation of tumour suppressor miRNAs suppressed the expression of oncogenes, which may be related to the recovery of drug sensitivity. ${ }^{71}$ Therefore, the selection of specific functional ncRNAs can be used as a new therapeutic option to promote prognosis and improve patient survival, reduce the level of drug resistance in cancer cells, and provide inspiration for finding new therapeutic targets. ${ }^{72}$

\section{MiRNAs Affect the Sensitivity of Chemotherapy for NPC}

miR-203 interacts with ZBE2, which binds directly to the miR-203 promoter and antagonizes miR-203, increasing cell migration, invasion, stemness and drug resistance; thus, overexpression of miR-203 can promote chemosensitivity. ${ }^{73}$ Overexpression of C-myc activates the EMT program and induces the CSC phenotype, therefore promoting drug sensitivity, while miR-200c can negatively feedback with $\mathrm{C}$-myc and inhibit $\mathrm{C}$-myc function, thus promoting chemoresistance. ${ }^{74}$ Moreover, Shao et al showed that overexpression of miR-139-5p reversed the progression of EMT in some nasopharyngeal carcinoma cells and improved the chemosensitivity of HNE1 and HNE1/DDP human nasopharyngeal carcinoma cells. ${ }^{75}$ Overexpression of miRBARTs can potentiate the sensitivity to chemotherapeutic agents in some nasopharyngeal epithelial cells. ${ }^{76}$ Yang et al revealed for the first time that viral LMP1 triggers the PI3K/ Akt/FOXO3a pathway to induce human miR-21 expression, which subsequently decreases the expression of PDCD4 and Fas-L and results in chemoresistance in NPC cells. ${ }^{77}$ Zhang et al proved that miRNA-19b contributes to the sensitivity of nasopharyngeal carcinoma to the chemotherapeutic agent cisplatin by targeting $\mathrm{KRAS}^{78}$ In addition, Bcl-2 and Mcl1 are two apoptosis-related targets, and their high expression represents an enhanced antiapoptotic capacity. High levels of miR-29c can inhibit the expression of Bcl-2 and Mcl-1, thus promoting apoptosis and therefore enhancing the sensitivity of NPC cells to radiotherapy and to the chemotherapeutic drug cisplatin. ${ }^{79}$ Moreover, miR-132 could enhance the DPP chemosensitivity of nasopharyngeal carcinoma cells by negatively regulating FOXA1 in vitro and in vivo. These data showed that miR-132 is a potential therapeutic target for NPC. ${ }^{80}$ It has been reported that mTOR can be a target of miR-3188, and miR-3188 targeting of mTOR can enhance its own expression level and sensitize NPC cells to 5 -FU. ${ }^{81}$ In addition, a number of studies have demonstrated that a variety of miRNAs play chemosensitization roles in nasopharyngeal carcinoma (Table 2).

\section{Pathways by Which miRNAs Influence Chemotherapy Resistance in NPC}

MiRNAs can mediate chemotherapy resistance in nasopharyngeal carcinoma by interacting with EMT-inducing transcription factors. There are many factors associated with tumour chemoresistance, and EMT and tumour stemness signalling are among the most important factors involved. ${ }^{82-84}$ In recent studies, miR-203 has been found to be involved in the pathogenesis of a large number of tumours, including nasopharyngeal carcinoma, and plays an inhibitory role in tumours. Jiang et $\mathrm{al}^{73}$ confirmed that ZEB2 could induce EMT and tumour stemness, and miR203 inhibited tumour cell progression and improved sensitivity to cisplatin by targeting ZEB2. ${ }^{85,86}$ miR-200 has also been reported to regulate the expression of ZEB1 and ZEB2 to suppress the EMT program. ${ }^{87}$ The miR-200 family can be regulated by the p53 gene, thereby suppressing EMT and CSC phenotypes. ${ }^{88,89}$ miR-200c formed a negative feedback pathway with $\mathrm{C}$-myc, and low expression of C-myc or increased expression of miR-200c promoted chemoresistance. $^{74}$ miR-139-5p targets ZEB1 and thereby regulates EMT, inhibits nasopharyngeal carcinoma cell progression, and promotes chemosensitivity to cisplatin. ${ }^{75,90}$

In addition, miRNAs may influence chemotherapeutic sensitivity by regulating cell cycle progression. KRAS can act as a link between upstream and downstream signals and as a transmitter to relay extracellular signals to the nucleus. ${ }^{91}$ MiR-19b enhances the sensitivity of nasopharyngeal carcinoma cells to the chemotherapeutic agent cisplatin by targeting KRAS, which is involved in cell cycle regulation. ${ }^{78}$ Zhao et al showed that mTOR, PI3K and AKT are associated with autophagy and are also cell cycle factors. mTOR can inhibit the PI3K/AKT signalling pathway, thereby inhibiting the downstream cell cycle factors c-JUN and p-mTOR. miR-3188 inhibits cell cycle 
Table 2 ncRNAs Involved in Resistance to Chemotherapy

\begin{tabular}{|c|c|c|c|c|c|}
\hline miRNA & Expression & Drugs & Targets & Functional Regulation & References \\
\hline miR-203 & $\uparrow$ & Cisplatin & ZEB2 & $\uparrow$ Chemo-sensitivity & [73] \\
\hline miR-200c & $\uparrow$ & Cisplatin & c-Myc & $\downarrow$ Chemo-sensitivity & [74] \\
\hline miR-BARTs & $\uparrow$ & Cisplatin, doxorubicin & BRCAI & $\uparrow$ Chemo-sensitivity & [76] \\
\hline miR-2I & $\uparrow$ & Cisplatin & PDCD4, Fas-L & $\downarrow$ Chemo-sensitivity & [77] \\
\hline miRNA-19b & $\uparrow$ & Cisplatin & KRAS & $\uparrow$ Chemo-sensitivity & [78] \\
\hline miRNA-29c & $\uparrow$ & Cisplatin & Mcl-I, Bcl-2 & $\uparrow$ Chemo-sensitivity & [79] \\
\hline miR-139-5p & $\uparrow$ & Cisplatin & EMT & $\uparrow$ Chemo-sensitivity & [75] \\
\hline miRNA-I 32 & $\uparrow$ & Cisplatin & FOXAI & $\uparrow$ Chemo-sensitivity & [80] \\
\hline miR-34c & $\downarrow$ & Cisplatin & soX4 & $\downarrow$ Chemo-sensitivity & [126] \\
\hline miR-96-5p & $\uparrow$ & Cisplatin & CDKI & $\uparrow$ Chemo-sensitivity & [127] \\
\hline miR-I83 & $\uparrow$ & Cisplatin & MTAI & $\uparrow$ Chemo-sensitivity & {$[128]$} \\
\hline miR-634 & $\uparrow$ & Paclitaxel & $*$ & $\uparrow$ Chemo-sensitivity & [129] \\
\hline miR-I204 & $\uparrow$ & Paclitaxel & $*$ & $\uparrow$ Chemo-sensitivity & [130] \\
\hline miR-I278 & $\uparrow$ & Cisplatin & ATG2B & $\uparrow$ Chemo-sensitivity & {$[|3|]$} \\
\hline miR-29c & $\uparrow$ & Paclitaxel & ITGBI & $\uparrow$ Chemo-sensitivity & [132] \\
\hline miR-29a & $\uparrow$ & Paclitaxel & STAT3, Bcl-2 & $\uparrow$ Chemo-sensitivity & [133] \\
\hline miR-3।88 & $\uparrow$ & 5-FU & mTOR & $\uparrow$ Chemo-sensitivity & [134] \\
\hline Lnc CCATI & $\uparrow$ & Paclitaxel & $\mathrm{miR} / 8 \mathrm{la} / \mathrm{CPEB} 2$ & $\uparrow$ Chemo-sensitivity & {$[105]$} \\
\hline Lnc KCNQIOTI & $\uparrow$ & Cisplatin & miR-454/USP47 axis & $\downarrow$ Chemo-sensitivity & {$[100]$} \\
\hline Lnc SLC25A2I-ASI & $\uparrow$ & Multidrug & miR-324-3p/IL-6 Axis & $\downarrow$ Chemo-sensitivity & {$[101]$} \\
\hline Lnc TINCR & $\uparrow$ & Cisplatin & acetyl-CoA & $\downarrow$ Chemo-sensitivity & [135] \\
\hline Lnc DLEUI & $\uparrow$ & Cisplatin & $\mathrm{BIRC6}$ & $\downarrow$ Chemo-sensitivity & [95] \\
\hline Lnc HOXAII-AS & $\downarrow$ & Cisplatin & miR-454-3p/c-Met & $\uparrow$ Chemo-sensitivity & [96] \\
\hline Lnc C00346 & $\uparrow$ & Cisplatin & miR-342-5p & $\downarrow$ Chemo-sensitivity & {$[102]$} \\
\hline Lnc MIAT & $\uparrow$ & Cisplatin & HMGBI & $\downarrow$ Chemo-sensitivity & [98] \\
\hline Lnc NEATI & $\downarrow$ & Cisplatin & Let-7a-5p & $\uparrow$ Chemo-sensitivity & [99] \\
\hline
\end{tabular}

Notes: $\uparrow$ Up-regulated and $\downarrow$ down-regulated ncRNAs in NPC, “*” target not specified.

signalling by targeting mTOR, PI3K/AKT and c-JUN, thereby enhancing the sensitivity of nasopharyngeal carcinoma to the chemotherapeutic agent 5-FU. ${ }^{81}$

The pathway by which miRNAs mediate chemotherapy sensitivity in nasopharyngeal carcinoma is also associated with apoptosis. Zhang et al showed that Bcl-2 and Mcl-1 are important antiapoptotic genes, and the authors found that their expression levels were negatively correlated with those of miR-29c. Loss of miR-29c function may lead to increased expression of NPC Bcl-2 and Mcl-1, and overexpression of miR-29c after cisplatin induction could promote nasopharyngeal carcinoma cell apoptosis and enhance the sensitivity of nasopharyngeal carcinoma to the chemotherapeutic drug cisplatin. ${ }^{79}$

\section{LncRNAs Affect the Chemosensitivity of NPC}

Increasing evidence has indicated that abnormal lncRNAs might lead to drug resistance in various types of cancer, ${ }^{92-94}$ including NPC. ${ }^{95,96}$ Paclitaxel is a classic chemotherapeutic agent in cancer chemotherapy, but with prolonged use and increased dosing, patients develop varying degrees of resistance to the drug. Cell growth assays showed that NPC 5-8F cells are highly tumorigenic and metastatic, 6-10B is a low tumorigenic and minimally metastatic cell line, and low expression of lncRNA n375709 can promote the sensitivity of nasopharyngeal carcinoma cells to the chemotherapeutic drug paclitaxel. ${ }^{97}$ Zhu et al proved that the expression levels of IncRNA MIAT and HMGB1 were elevated in drug-resistant NPC cells, and IL6 could activate the JAK2/STAT3 signalling pathway, thus making the tumour resistant. HMGB1 could target IL6 and increase the expression level of IL6, thus promoting chemoresistance of the tumour, and IncRNA MIAT could positively regulate, thus forming the lncRNA MIAT/HMGB1/JAK2/STAT3 signalling axis, which plays a role in chemoresistance of tumours. Therefore, lncRNA MIAT can be used as a target, and inhibition of lncRNA MIAT expression can reverse chemoresistance in nasopharyngeal carcinoma to some extent. ${ }^{98}$ The authors found that lnc NEAT1 interacted with let-7a-5p, and the two were negatively correlated. Let-7a-5p inhibited chemoresistance and improved 
chemosensitivity in nasopharyngeal carcinoma. Overexpression of NEAT1 regulates Rsf-1 expression and the Ras-MAPK pathway by inhibiting let-7a-5p, thereby rendering nasopharyngeal carcinoma cells resistant to the chemotherapeutic agent cisplatin. ${ }^{99}$ Existing research results indicated that KcNQ1OT1 enhanced DDP resistance in NPC cells via the miR-454/USP47 axis, suggesting a potential therapeutic target for patients with DDP-resistant NPC. ${ }^{100}$ Wang et al demonstrated elevated expression levels of lncRNA SLC25A21-AS1 in NPC tissues and cells. In addition, high expression of SLC25A21-AS1 could target miR-324-3p and thus mediate IL-6, resulting in enhanced proliferation and multidrug resistance in NPC cells. ${ }^{101}$ It has been reported that lnc C00346 targets miR-342-5p and reduces the sensitivity of nasopharyngeal carcinoma cells to cisplatin. ${ }^{102}$ In addition, a variety of lncRNAs may be involved in the regulation of chemotherapy sensitivity in nasopharyngeal carcinoma (Table 2).

The existence of multiple lncRNAs that can inhibit or enhance sensitivity to radiotherapy and chemotherapy in nasopharyngeal carcinoma can serve as inspiration for the direction of treatment for drug-resistant patients. In future studies, we can find a breakthrough in reversing drug resistance by suppressing lncRNAs that are highly expressed in cells that develop drug resistance or upregulating lncRNAs that are expressed at low levels in drugsensitive cell lines to achieve the desired therapeutic effect and patient survival. ${ }^{103}$ This study provides a new idea and a new direction for reversing chemotherapy resistance in nasopharyngeal carcinoma.

\section{Pathways by Which IncRNAs Regulate Chemotherapy Resistance in NPC}

After a long period of research and exploration, the following mechanisms can be identified for lncRNAs to regulate drug resistance in tumours: directly targeting miRNAs or interacting with miRNAs, mediating signalling pathways and regulating protein function, inhibiting or enhancing apoptosis, and regulating autophagy. ${ }^{104}$ In nasopharyngeal carcinoma, lncRNAs mainly exert their influence by regulating miRNAs and targeting signalling pathways and apoptosis-related proteins, among which various regulatory pathways also interact with each other.

LncRNAs can target miRNA-related signalling axes or interact with miRNAs to regulate chemotherapy sensitivity in nasopharyngeal carcinoma. Yuan et al suggested that IncRNA KcNQ1OT1 facilitated cell viability and DDP resistance in NPC cells by regulating the miR-454/USP47 axis. Therefore, KcNQ1OT1 might serve as a potential target for overcoming DDP resistance in NPC chemotherapy. ${ }^{100}$ Wang et al showed that miR-181a could enhance the sensitivity of nasopharyngeal carcinoma cells to paclitaxel by promoting apoptosis, while in nasopharyngeal carcinoma, IncCCAT 1 could directly bind to miR-181a, thus inhibiting the biological function of miR-181a and making nasopharyngeal carcinoma cells less sensitive to paclitaxel. ${ }^{105}$ In addition, Inc SLC25A21-AS1 regulates IL-6 by targeting miR-324-3p, making nasopharyngeal carcinoma resistant to multiple chemotherapeutic agents. ${ }^{101} \mathrm{Li}$ et al revealed that lnc DLEU1 inhibits miR-381-3p, resulting in increased expression of BIRC6 and increased resistance of nasopharyngeal carcinoma to the chemotherapeutic agent cisplatin. ${ }^{95}$ Lin et al demonstrated that silencing lncRNA HOXA11-AS can inhibit the c-Met/AKT/mTOR pathway by specifically upregulating miR-454-3p, thus promoting cell apoptosis and enhancing the sensitivity of cisplatin-resistant NPC cells to cisplatin. ${ }^{96}$ Long noncoding RNA LINC00346 contributes to cisplatin resistance in nasopharyngeal carcinoma by repressing miR-342-5p. ${ }^{102}$

In addition, IncRNAs can also influence drug resistance in nasopharyngeal carcinoma by mediating signalling pathways. Zhu et al proved that lncRNAs MIAT and HMGB1 are upregulated in cisplatin-resistant nasopharyngeal carcinoma cells, are biological targets that promote cisplatin resistance, and can initiate the JAK2/STAT3 pathway by promoting the expression level of self-IL6, further increasing cisplatin resistance in nasopharyngeal carcinoma cells. ${ }^{98}$ Moreover, Ras-MAPK is an important intracellular signalling pathway that regulates apoptosis, the cell cycle and many other cellular processes. Low expression of NEAT1 inhibited the expression level of let-7a-5p, which reduced the activity of the Ras-MAPK signalling pathway and increased the sensitivity of NPC cells to cisplatin. $^{99}$

In tumours, proapoptotic and antiapoptotic proteins regulate each other to stabilize the intratumour environment, with Bcl-2 being the classical antiapoptotic protein. The apoptotic ability of cells is a key factor in assessing the effect of tumour chemotherapy, and chemotherapeutic drugs can mediate apoptosis-related proteins and promote apoptosis to achieve a desirable chemotherapeutic effect. ${ }^{106}$ Thus, low expression of the antiapoptotic protein Bcl-2 can promote tumour sensitivity to chemotherapeutic agents. ${ }^{107}$ Xue et al found that low expression of IncRNAROR can promote apoptosis in NPC cells, indicating that 
we can use inhibitors of lncRNA-ROR to promote apoptosis and restore the sensitivity of nasopharyngeal carcinoma to chemotherapy. ${ }^{108}$

\section{Conclusions and Future Perspectives}

The World Health Organization classifies nasopharyngeal carcinoma into three pathological subtypes: keratinizing squamous carcinoma, non-keratinizing squamous carcinoma, and basal-like squamous carcinoma. Nonkeratinizing nasopharyngeal carcinoma can be divided into differentiated and undifferentiated tumours, and the nonkeratinizing subtype associated with EB virus (EBV) infection constitutes the majority of cases $(>95 \%)$ in endemic areas. Therefore, this type deserves our focused attention. In this article, the effects and mechanisms of miRNAs and IncRNAs in the resistance to radiotherapy and chemotherapy in nasopharyngeal carcinoma are summarized and analyzed. miRNAs can affect tumour radioresistance by interfering with multiple pathways. In addition, a large number of studies have shown that miRNAs can affect chemotherapy resistance in nasopharyngeal carcinoma by regulating apoptosis, cell cycle and EMT-related signalling. We speculate that the same regulatory pathway may exist between radiotherapy resistance and chemotherapy resistance in nasopharyngeal carcinoma, or that there may be miRNAs that can affect both radiotherapy and chemotherapy in nasopharyngeal carcinoma: this remains to be investigated. Interestingly, miR-206 and miR-451 and multiple miRNAs were found to be downregulated in a variety of cancers other than nasopharyngeal carcinoma. This suggests that we can be inspired by key molecules already identified in other cancers to find key therapeutic targets for nasopharyngeal carcinoma. In current studies, lncRNAs regulate the radiosensitivity of nasopharyngeal carcinoma mainly by mediating DNA damage, regulating the cell cycle, and targeting miRNAs. LncRNAs mainly play a role in regulating the effects of chemotherapy resistance in nasopharyngeal carcinoma by regulating miRNAs, targeting signalling pathways and apoptosis-related proteins, in which various regulatory pathways also interact with each other. This suggests that lncRNAs can interact with miRNAs through multiple pathways and molecules, and that there may be common regulatory pathways.

LncRNAs and miRNAs contained in ncRNAs have powerful biological functions. In nasopharyngeal carcinoma cells resistant to radiotherapy, elevated expression levels of some lncRNAs and miRNAs can be found, and interestingly, most of these lncRNAs and miRNAs are silently expressed in cells sensitive to radiotherapy. We can speculate that inhibiting their expression in resistant cells or upregulating lncRNAs and miRNAs with functions related to promoting apoptosis and inhibiting proliferation and metastasis can reverse the radiation resistance. Most of the current data on ncRNAs related to chemoresistance and radioresistance in nasopharyngeal carcinoma focus on miRNAs and lncRNAs, which indicates that miRNAs and lncRNAs have been studied in some contexts, but that circRNAs, which are included among ncRNAs, have not been fully studied in nasopharyngeal carcinoma at present because of their numerous types and complex functions. circRNAs can act as ceRNAs for miRNAs and disrupt the inhibitory effects of miRNAs on target genes, thus exerting their anticancer or cancerpromoting effects. There are fewer studies on circRNAs affecting tumour progression through other pathways and molecular mechanisms, which is a possible breakthrough point for future treatment and prognosis improvement in nasopharyngeal carcinoma, thus developing individualized treatment for nasopharyngeal carcinoma patients and benefiting more patients. Current research data demonstrate that miRNAs and lncRNAs are useful biomarkers for predicting treatment outcomes or monitoring treatment response, but most studies are still in the preclinical stage and have not been clinically tested. In addition, only a small fraction of ncRNAs are stably present in body fluids, so future studies could use non-invasive liquid biopsy methods. It is noteworthy that some studies assessing the potential of ncRNAs as biomarkers in different cancers have yielded conflicting results. More clinical data should be mined to reconcile these controversies.

It is hoped that in the near future, specific ncRNA signalling may provide new insights into the mechanisms of chemotherapy and radiation resistance that may arise in nasopharyngeal carcinoma patients before starting treatment, while the regulation of specific ncRNA expression may provide new tools for overcoming acquired resistance. In conclusion, the identification of candidate non-coding RNAs that regulate drug resistance in nasopharyngeal carcinoma and the study of their molecular mechanisms can help aid the design of new and targeted non-coding RNA-based therapeutic strategies and provide new ideas for improving clinical outcomes in patients with nasopharyngeal carcinoma. miRNAs and lncRNAs are considered to have considerable clinical value in oncology, and their potential clinical application is of great significance. 


\section{Disclosure}

The authors report no conflicts of interest in this work.

\section{References}

1. Chen Y-P, Chan ATC, Le Q-T, Blanchard P, Sun Y, Ma J. Nasopharyngeal carcinoma. Lancet. 2019;394(10192):64-80. doi:10.1016/S0140-6736(19)30956-0

2. Sun X-S, Li X-Y, Chen Q-Y, Tang L-Q, Mai H-Q. Future of radiotherapy in nasopharyngeal carcinoma. BJR. 2019;92 (1102):20190209. doi:10.1259/bjr.20190209

3. Perri F, Della Vittoria Scarpati G, Caponigro F, et al. Management of recurrent nasopharyngeal carcinoma: current perspectives. OTT. 2019;12:1583-1591. doi:10.2147/OTT.S188148

4. Wang C, Yang Y, Sun L, et al. Baicalin reverses radioresistance in nasopharyngeal carcinoma by downregulating autophagy. Cancer Cell Int. 2020;20(1):35. doi:10.1186/s12935-020-1107-4

5. Guan S, Wei J, Huang L, Wu L. Chemotherapy and chemo-resistance in nasopharyngeal carcinoma. Eur J Med Chem. 2020;207:112758. doi:10.1016/j.ejmech.2020.112758

6. Haider T, Pandey V, Banjare N, Gupta PN, Soni V. Drug resistance in cancer: mechanisms and tackling strategies. Pharmacol Rep. 2020;72(5):1125-1151. doi:10.1007/s43440-020-00138-7

7. Bukowski K, Kciuk M, Kontek R. Mechanisms of multidrug resistance in cancer chemotherapy. IJMS. 2020;21(9):3233. doi:10.3390/ijms21093233

8. Luo G, Jin K, Deng S, et al. Roles of CA19-9 in pancreatic cancer: biomarker, predictor and promoter. Biochimica Et Biophysica Acta (BBA) - Rev Cancer. 2021;1875(2):188409. doi:10.1016/j.bbcan.2020.188409

9. Bautista-Sánchez D, Arriaga-Canon C, Pedroza-Torres A, et al. The promising role of miR-21 as a cancer biomarker and its importance in RNA-based therapeutics. Mol Ther - Nucleic Acids. 2020;20:409-420. doi:10.1016/j.omtn.2020.03.003

10. Lei F, Lei T, Huang Y, Yang M, Liao M, Huang W. RadioSusceptibility of Nasopharyngeal Carcinoma: Focus on EpsteinBarr Virus, MicroRNAs, Long Non-Coding RNAs and Circular RNAs. CMP. 2020;13(3):192-205. doi:10.2174/ 1874467213666191227104646.

11. Guan Y, Zhang M, Chen X, Zhang Q, Liu S, Lnc ZY. RNA SNHG20 participated in proliferation, invasion, and migration of breast cancer cells via miR-495. J Cell Biochem. 2018;119 (10):7971-7981. doi:10.1002/jcb.26588

12. Yu W, Sun Z, Yang L, et al. IncRNA PTAR promotes NSCLC cell proliferation, migration and invasion by sponging microRNA-101. Mol Med Rep. 2019. doi:10.3892/ mmr.2019.10646

13. Song W, Wang J, Liu H, et al. Effects of LncRNA Lnc-LIF-AS on cell proliferation, migration and invasion in a human cervical cancer cell line. Cytokine. 2019;120:165-175. doi:10.1016/j. cyto.2019.05.004

14. Bayraktar R, Van Roosbroeck K. miR-155 in cancer drug resistance and as target for miRNA-based therapeutics. Cancer Metastasis Rev. 2018;37(1):33-44. doi:10.1007/s10555-017-9724-7

15. Ebahimzadeh K, Shoorei H, Mousavinejad SA, et al. Emerging role of non-coding RNAs in response of cancer cells to radiotherapy. Pathol - Res Pract. 2021;218:153327. doi:10.1016/ j.prp.2020.153327

16. Tian Y, Tang L, Yi P, et al. MiRNAs in radiotherapy resistance of nasopharyngeal carcinoma. J Cancer. 2020;11(13):3976-3985. doi: $10.7150 /$ jca. 42734

17. Wang S, Zhang R, Claret FX, Yang H. Involvement of microRNA-24 and DNA methylation in resistance of nasopharyngeal carcinoma to ionizing radiation. Mol Cancer Ther. 2014;13(12):3163-3174. doi:10.1158/1535-7163.MCT-14-0317
18. Huang T, Yin L, Wu J, et al. MicroRNA-19b-3p regulates nasopharyngeal carcinoma radiosensitivity by targeting TNFAIP3/NFкB axis. J Exp Clin Cancer Res. 2016;35(1):188. doi:10.1186/ s13046-016-0465-1

19. Bao B, Li Y, Ahmad A, et al. Targeting CSC-related miRNAs for cancer therapy by natural agents. CDT. 2012;13(14):1858-1868. doi:10.2174/138945012804545515

20. Barker HE, Paget JTE, Khan AA, Harrington KJ. The tumour microenvironment after radiotherapy: mechanisms of resistance and recurrence. Nat Rev Cancer. 2015;15(7):409-425. doi: $10.1038 / \mathrm{nrc} 3958$

21. Doronkin S, Djagaeva I, Beckendorf SK. CSN5/Jab1 mutations affect axis formation in the Drosophila oocyte by activating a meiotic checkpoint. 12 .

22. Su M, Wang H, Wang W, et al. LncRNAs in DNA damage response and repair in cancer cells. Acta Biochim Biophys Sin (Shanghai). 2018;50(5):433-439. doi:10.1093/abbs/gmy022

23. Nie X, Guo E, Wu C, et al. SALL4 induces radioresistance in nasopharyngeal carcinoma via the ATM/Chk2/p53 pathway. Cancer Med. 2019;8(4):1779-1792. doi:10.1002/cam4.2056

24. Hu X, Jiang H, Jiang X. Downregulation of lncRNA ANRIL inhibits proliferation, induces apoptosis, and enhances radiosensitivity in nasopharyngeal carcinoma cells through regulating miR-125a. Cancer Biol Ther. 2017;18(5):331-338. doi:10.1080/ 15384047.2017.1310348

25. Manvati MKS, Khan J, Verma N, Dhar PK. Association of miR-760 with cancer: an overview. Gene. 2020;747:144648. doi:10.1016/j.gene.2020.144648

26. Zhang T, Sun Q, Liu T, et al. MiR-451 increases radiosensitivity of nasopharyngeal carcinoma cells by targeting ras-related protein 14 (RAB14). Tumor Biol. 2014;35(12):12593-12599. doi:10.1007/s13277-014-2581-x

27. Wang T, Dong X-M, Zhang F-L, Zhang J-R. miR-206 enhances nasopharyngeal carcinoma radiosensitivity by targeting IGF1. Kaohsiung J Med Sci. 2017;33(9):427-432. doi:10.1016/j. kjms.2017.05.015

28. Nohata N, Hanazawa T, Enokida H, Seki N. microRNA-1/133a and microRNA-206/133b clusters: dysregulation and functional roles in human cancers. Oncotarget. 2012;3:1. doi:10.18632/ oncotarget. 424

29. Chen QY, Jiao DM, Wang J, Hu H, Lu W. miR-206 regulates cisplatin resistance and EMT in human lung adenocarcinoma cells partly by targeting MET. Oncotarget. 2016;7(17):24510-24526. doi:10.18632/oncotarget.8229

30. Zhang Y, Zheng L, Lin S, Liu Y, Wang Y, Gao F. MiR-124 enhances cell radiosensitivity by targeting PDCD6 in nasopharyngeal carcinoma. Int J Clin Exp Pathol. 2017;10(12):11461.

31. Guo Y, Zhai J, Zhang J, Ni C, Zhou H. Improved radiotherapy sensitivity of nasopharyngeal carcinoma cells by miR-29-3p targeting COL1A1 3'-UTR. Med Sci Monit. 2019;25:3161-3169. doi:10.12659/MSM.915624

32. Kong L, Wei Q, Hu X, Chen L, Li J. miR-193a-3p promotes radio-resistance of nasopharyngeal cancer cells by targeting SRSF2 gene and hypoxia signaling pathway. Med Sci Monit Basic Res. 2019;25:53-62. doi:10.12659/MSMBR.914572

33. Huang Y, Tan D, Xiao J, Li Q, Zhang X, Luo Z. miR-150 contributes to the radioresistance in nasopharyngeal carcinoma cells by targeting glycogen synthase kinase-3 $\beta$. J Can Res Ther. 2018;14(1):111. doi:10.4103/jcrt.JCRT_682_17

34. He W, Jin H, Liu Q, Sun Q. miR-182-5p contributes to radioresistance in nasopharyngeal carcinoma by regulating BNIP3 expression. Mol Med Rep. 2020;23(2):130. doi:10.3892/mmr.2020.11769

35. Wang F, Lu J, Peng X, et al. Integrated analysis of microRNA regulatory network in nasopharyngeal carcinoma with deep sequencing. J Exp Clin Cancer Res. 2016;35(1):17. doi:10.1186/ s13046-016-0292-4 
36. Guo P, Lan J, Ge J, et al. MiR-26a enhances the radiosensitivity of glioblastoma multiforme cells through targeting of ataxiatelangiectasia mutated. Exp Cell Res. 2014;320(2):200-208. doi:10.1016/j.yexcr.2013.10.020

37. Liu S, Pan X, Yang Q, et al. MicroRNA-18a enhances the radiosensitivity of cervical cancer cells by promoting radiation-induced apoptosis. Oncol Rep. 2015;33(6):2853-2862. doi:10.3892/ or.2015.3929

38. Wang P, Zou F, Zhang X, et al. microRNA-21 negatively regulates $\mathrm{Cdc} 25 \mathrm{~A}$ and cell cycle progression in colon cancer cells. Cancer Res. 2009;69(20):8157-8165. doi:10.1158/0008-5472. CAN-09-1996

39. Tang Y, Cui Y, Li Z, et al. Radiation-induced miR-208a increases the proliferation and radioresistance by targeting p21 in human lung cancer cells. J Exp Clin Cancer Res. 2016;35(1):7. doi:10.1186/s13046-016-0285-3

40. Chen G, Zhu W, Shi D, et al. MicroRNA-181a sensitizes human malignant glioma U87MG cells to radiation by targeting Bcl-2. Oncol Rep. 2010;23:4. doi:10.3892/or_00000725

41. Edison N, Curtz Y, Paland N, et al. Degradation of Bcl-2 by XIAP and ARTS promotes apoptosis. Cell Rep. 2017;21(2):442-454. doi:10.1016/j.celrep.2017.09.052

42. Cory S, Adams JM. The Bcl2 family: regulators of the cellular life-or-death switch. Nat Rev Cancer. 2002;2(9):647-656. doi: $10.1038 / \mathrm{nrc} 883$

43. Qu C, Liang Z, Huang J, et al. MiR-205 determines the radioresistance of human nasopharyngeal carcinoma by directly targeting PTEN. Cell Cycle. 2012;11(4):785-796. doi:10.4161/ cc.11.4.19228

44. Mao Y, Wu S, Zhao R, Deng Q. MiR-205 promotes proliferation, migration and invasion of nasopharyngeal carcinoma cells by activation of AKT signalling. $J$ Int Med Res. 2016;44 (2):231-240. doi:10.1177/0300060515576556

45. Ou H, Li Y, Kang M. Activation of miR-21 by STAT3 induces proliferation and suppresses apoptosis in nasopharyngeal carcinoma by targeting PTEN gene. PLoS One. 2014;9(11):e109929. doi:10.1371/journal.pone.0109929

46. Ciccia A, Elledge SJ. The DNA damage response: making it safe to play with knives. Mol Cell. 2010;40(2):179-204. doi:10.1016/j. molcel.2010.09.019

47. Lung RW-M, Hau P-M, Yu KH-O, et al. EBV-encoded miRNAs target ATM-mediated response in nasopharyngeal carcinoma: regulation of ATM by EBV-miRNAs in NPC. $J$ Pathol. 2018;244(4):394-407. doi:10.1002/path.5018

48. Pan Y, Zhang Q, Atsaves V, Yang H, Claret FX. Suppression of Jab1/CSN5 induces radio- and chemo-sensitivity in nasopharyngeal carcinoma through changes to the DNA damage and repair pathways. Oncogene. 2013;32(22):2756-2766. doi:10.1038/ onc.2012.294

49. Pan Y, Zhang Q, Tian L, et al. Jab1/CSN5 negatively regulates p27 and plays a role in the pathogenesis of nasopharyngeal carcinoma. Cancer Res. 2012;72(7):1890-1900. doi:10.1158/ 0008-5472.CAN-11-3472

50. Wu J, Lv Q, He J, et al. MicroRNA-188 suppresses G1/S transition by targeting multiple cyclin/CDK complexes. Cell Commun Signaling. 2014;12:1-3.

51. Lu J, He M-L, Wang L, et al. MiR-26a inhibits cell growth and tumorigenesis of nasopharyngeal carcinoma through repression of EZH2. Cancer Res. 2011;71(1):225-233. doi:10.1158/0008-5472. CAN-10-1850

52. Liang S, Zhang N, Deng Y, et al. miR-663 promotes NPC cell proliferation by directly targeting CDKN2A. Mol Med Rep. 2017;16(4):4863-4870. doi:10.3892/mmr.2017.7129

53. Autuoro J, Pirnie S, Carmichael G. Long noncoding RNAs in imprinting and X chromosome inactivation. Biomolecules. 2014;4 (1):76-100. doi:10.3390/biom4010076
54. Di Gesualdo F, Capaccioli S, Lulli M. A pathophysiological view of the long non-coding RNA world. Oncotarget. 2014;5 (22):10976-10996. doi:10.18632/oncotarget.2770

55. Yang-Q-Q, Deng Y-F. Genome-wide analysis of long non-coding RNA in primary nasopharyngeal carcinoma by microarray. Histopathology. 2015;66(7):1022-1030. doi:10.1111/his.12616

56. Schmitt AM, Chang HY. Long RNAs wire up cancer growth. Nature. 2013;500(7464):536-537. doi:10.1038/nature12548

57. Nie Y, Liu X, Qu S, Song E, Zou H, Gong C. Long non-coding RNA HOTAIR is an independent prognostic marker for nasopharyngeal carcinoma progression and survival. Cancer Sci. 2013;104 (4):458-464. doi:10.1111/cas.12092

58. Han Q, Li L, Liang H, Li Y, Xie J, Wang Z. Downregulation of lncRNA $\mathrm{X}$ inactive specific transcript (XIST) suppresses cell proliferation and enhances radiosensitivity by upregulating mir-29c in nasopharyngeal carcinoma cells. Med Sci Monit. 2017;23:4798-4807. doi:10.12659/MSM.905370

59. Ma X, Zhou J, Liu J, et al. LncRNA ANCR promotes proliferation and radiation resistance of nasopharyngeal carcinoma by inhibiting PTEN expression. OTT. 2018;11:8399-8408. doi:10.2147/OTT.S182573

60. Zhong Q, Chen Y, Chen Z. LncRNA MINCR regulates irradiation resistance in nasopharyngeal carcinoma cells via the microRNA-223/ZEB1 axis. Cell Cycle. 2020;19(1):53-66. doi:10.1080/15384101.2019.1692176

61. He Y, Jing Y, Wei F, et al. Long non-coding RNA PVT1 predicts poor prognosis and induces radioresistance by regulating DNA repair and cell apoptosis in nasopharyngeal carcinoma. Cell Death Dis. 2018;9(2):235. doi:10.1038/s41419-018-0265-y

62. Wang Y, Chen W, Lian J, et al. The IncRNA PVT1 regulates nasopharyngeal carcinoma cell proliferation via activating the KAT2A acetyltransferase and stabilizing HIF-1 $\alpha$. Cell Death Differ. 2020;27(2):695-710. doi:10.1038/s41418-019-0381-y

63. Wang Q, Fan H, Liu Y, et al. Curcumin enhances the radiosensitivity in nasopharyngeal carcinoma cells involving the reversal of differentially expressed long non-coding RNAs. Int J Oncol. 2014;44(3):858-864. doi:10.3892/ijo.2013.2237

64. Zhang Z, Zhu Z, Watabe K, et al. Negative regulation of lncRNA GAS5 by miR-21. Cell Death Differ. 2013;20(11):1558-1568. doi: $10.1038 /$ cdd.2013.110

65. Liu X, Sun M, Nie F, et al. Lnc RNA HOTAIR functions as a competing endogenous RNA to regulate HER2 expression by sponging miR-331-3p in gastric cancer. Mol Cancer. 2014;13 (1):92. doi:10.1186/1476-4598-13-92

66. Han YY, Liu K, Xie J, Li F, Wang Y, Yan B. LINC00114 promoted nasopharyngeal carcinoma progression and radioresistance in vitro and in vivo through regulating ERK/JNK signaling pathway via targeting miR-203. Eur Rev Med Pharmacol Sci. 2020;24:2491-2504.

67. Huang D, Zhu X, Wang Y, Yu H, Pu Y. Long non-coding RNA FAM133B-2 represses the radio-resistance of nasopharyngeal cancer cells by targeting miR-34a-5p/CDK6 axis. Aging. 2020;12(17):16936-16950. doi:10.18632/aging.103600

68. Lu Y, Li T, Wei G, et al. The long non-coding RNA NEAT1 regulates epithelial to mesenchymal transition and radioresistance in through miR-204/ZEB1 axis in nasopharyngeal carcinoma. Tumor Biol. 2016;37(9):11733-11741. doi:10.1007/s13277-015-4773-4

69. Paiar F, Di Cataldo V, Zei G, et al. Role of chemotherapy in nasopharyngeal carcinoma. Oncol Rev. 2012;6(1):1. doi:10.4081/ oncol.2012.e1

70. Zheng T, Wang J, Chen X, Liu L. Role of microRNA in anticancer drug resistance. Int $J$ Cancer. 2010;126(1):2-10. doi: $10.1002 / \mathrm{ijc} .24782$

71. Donzelli S, Mori F, Biagioni F, et al. MicroRNAs: short non-coding players in cancer chemoresistance. Mol and Cell Ther. 2014;2(1):16. doi:10.1186/2052-8426-2-16 
72. Hummel R, Hussey DJ, Haier J. MicroRNAs Predictors and modifiers of chemo- and radiotherapy in different tumour types. pdf. Eur J Cancer. 2010;46:298-311.

73. Jiang $\mathrm{Q}$, Zhou $\mathrm{Y}$, Yang $\mathrm{H}$, et al. A directly negative interaction of miR-203 and ZEB2 modulates tumor stemness and chemotherapy resistance in nasopharyngeal carcinoma. Oncotarget. 2016;7 (41):67288-67301. doi:10.18632/oncotarget.11691

74. Yang J, Wu S-P, Wang W-J, et al. A novel miR-200c/c-myc negative regulatory feedback loop is essential to the EMT process, CSC biology and drug sensitivity in nasopharyngeal cancer. Exp Cell Res. 2020;391(2):111817. doi:10.1016/j.yexcr.2020.111817

75. Shao Q, Zhang P, Ma Y, et al. MicroRNA-139-5p affects cisplatin sensitivity in human nasopharyngeal carcinoma cells by regulating the epithelial-to-mesenchymal transition. Gene. 2018;652:48-58. doi:10.1016/j.gene.2018.02.003

76. Lung RW, Tong JH, Ip L, et al. EBV-encoded miRNAs can sensitize nasopharyngeal carcinoma to chemotherapeutic drugs by targeting BRCA1. J Cell Mol Med. 2020;24 (22):13523-13535. doi:10.1111/jcmm.16007

77. Yang G-D, Huang T-J, Peng L-X, et al. Epstein-Barr virus encoded LMP1 upregulates MicroRNA-21 to promote the resistance of nasopharyngeal carcinoma cells to cisplatin-induced apoptosis by suppressing PDCD4 and Fas-L. PLoS One. 2013;8(10): e78355. doi:10.1371/journal.pone. 0078355

78. Zhang Y, Zhao Y, Liu L, et al. MicroRNA-19b promotes nasopharyngeal carcinoma more sensitive to cisplatin by suppressing KRAS. Technol Cancer Res Treat. 2018;17:153303381879365. doi:10.1177/1533033818793652

79. Zhang J-X, Qian D, Wang F-W, et al. MicroRNA-29c enhances the sensitivities of human nasopharyngeal carcinoma to cisplatin-based chemotherapy and radiotherapy. Cancer Lett. 2013;329(1):91-98. doi:10.1016/j.canlet.2012.10.033

80. Li YL, Zhao YG, Chen B, Li XF. MicroRNA-132 sensitizes nasopharyngeal carcinoma cells to cisplatin through regulation of forkhead box A1 protein. Pharmazie. 2016;12:715-718. doi:10.1691/ph.2016.6764

81. Zhao M, Luo R, Kim M, Do J, Lee D, Han H. miR-3188 regulates nasopharyngeal carcinoma proliferation and chemosensitivity through a FOXO1-modulated positive feedback loop with mTOR-p-PI3K/AKT-c-JUN. Polym J. 2016;48(7):829-834. doi:10.1038/pj.2016.37

82. Zhu P, Wang Y, Du Y, et al. C8orf4 negatively regulates self-renewal of liver cancer stem cells via suppression of NOTCH2 signalling. Nat Commun. 2015;6(1):7122. doi:10.1038/ncomms8122

83. Lei Z-J, Wang J, Xiao H-L, et al. Lysine-specific demethylase 1 promotes the stemness and chemoresistance of Lgr5+ liver cancer initiating cells by suppressing negative regulators of $\beta$-catenin signaling. Oncogene. 2015;34(24):3188-3198. doi:10.1038/onc.2015.129

84. Koo BS, Lee SH, Kim JM, et al. Oct4 is a critical regulator of stemness in head and neck squamous carcinoma cells. Oncogene. 2015;34(18):2317-2324. doi:10.1038/onc.2014.174

85. Wang L, Tian W-D, Xu X, et al. Epstein-Barr virus nuclear antigen 1 (EBNA1) protein induction of epithelial-mesenchymal transition in nasopharyngeal carcinoma cells: EBNA1 Induces EMT and Metastasis. Cancer. 2014;120(3):363-372. doi: $10.1002 /$ cncr. 28418

86. Xia H, Cheung WKC, Sze J, et al. miR-200a regulates epithelialmesenchymal to stem-like transition via ZEB2 and $\beta$-catenin signaling. $J$ Biol Chem. 2010;285(47):36995-37004. doi:10.1074/jbc.M110.133744

87. Radisky DC. miR-200c at the nexus of epithelial-mesenchymal transition, resistance to apoptosis, and the breast cancer stem cell phenotype. Breast Cancer Res. 2011;13(3):110, bcr2885. doi: $10.1186 / \mathrm{bcr} 2885$
88. Chang C-J, Chao C-H, Xia W, et al. p53 regulates epithelialmesenchymal transition and stem cell properties through modulating miRNAs. Nat Cell Biol. 2011;13(3):317-323. doi:10.1038/ ncb2173

89. Kim T, Veronese A, Pichiorri F, et al. p53 regulates epithelialmesenchymal transition through microRNAs targeting ZEB1 and ZEB2. J Exp Med. 2011;208(5):875-883. doi:10.1084/jem.20110235

90. Yue S, Wang L, Zhang H, et al. miR-139-5p suppresses cancer cell migration and invasion through targeting ZEB1 and ZEB2 in GBM. Tumor Biol. 2015;36(9):6741-6749. doi:10.1007/s13277015-3372-8

91. Zuber J, Tchernitsa OI, Hinzmann B, et al. A genome-wide survey of RAS transformation targets. Nat Genet. 2000;24 (2):144-152. doi:10.1038/72799

92. Hu M, Zhang Q, Tian X, Wang J, Niu Y, Li G. IncRNA CCAT1 is a biomarker for the proliferation and drug resistance of esophageal cancer via the miR-143/PLK1/BUBR1 axis. Mol Carcinog. 2019;58(12):2207-2217. doi:10.1002/mc.23109

93. Fu D, Lu C, Qu X, et al. LncRNA TTN-AS1 regulates osteosarcoma cell apoptosis and drug resistance via the miR-134-5p/ MBTD1 axis. Aging. 2019;11(19):8374-8385. doi:10.18632/ aging. 102325

94. Zhuang J, Shen L, Yang L, et al. TGF $\beta 1$ promotes gemcitabine resistance through regulating the LncRNA-LET/NF90/miR-145 signaling axis in bladder cancer. Theranostics. 2017;7 (12):3053-3067. doi:10.7150/thno.19542

95. Li H, Huang J, Yu S, Lou Z. Long non-coding RNA DLEU1 up-regulates BIRC6 expression by competitively sponging miR-381-3p to promote cisplatin resistance in nasopharyngeal carcinoma. OTT. 2020;13:2037-2045. doi:10.2147/OTT.S237456

96. Lin F-J, Lin X-D, Xu L-Y, Zhu S-Q. Long noncoding RNA HOXA11-AS modulates the resistance of nasopharyngeal carcinoma cells to cisplatin via miR-454-3p/c-Met. Mol Cells. 2020;43:856.

97. Ren S, Li G, Liu C, et al. Next generation deep sequencing identified a novel lncRNA n375709 associated with paclitaxel resistance in nasopharyngeal carcinoma. Oncol Rep. 2016;36 (4):1861-1867. doi:10.3892/or.2016.4981

98. Zhu X, Liu L, Wang Y, et al. IncRNA MIAT/HMGB1 axis is involved in cisplatin resistance via regulating IL6-mediated activation of the JAK2/STAT3 pathway in nasopharyngeal carcinoma. Front Oncol. 2021;11:651693. doi:10.3389/ fonc. 2021.651693

99. Liu F, Tai Y, Ma J. LncRNA NEAT1/let-7a-5p axis regulates the cisplatin resistance in nasopharyngeal carcinoma by targeting Rsf-1 and modulating the Ras-MAPK pathway. Cancer Biol Ther. 2018;19(6):534-542. doi:10.1080/15384047.2018.1450119

100. Yuan F, Lou Z, Zhou Z, Yan X. Long non-coding RNA KCNQ1OT1 promotes nasopharyngeal carcinoma cell cisplatin resistance via the miR-454/USP47 axis. Int J Mol Med. 2021;47 (4):54. doi:10.3892/ijmm.2021.4887

101. Wang $\mathrm{X}$, Wang $\mathrm{C}, \mathrm{Xu} \mathrm{H}$, Xie $\mathrm{H}$. Long non-coding RNA SLC25A21-AS1 promotes multidrug resistance in nasopharyngeal carcinoma by regulating miR-324-3p/IL-6 axis. CMAR. 2020;12:3949-3957. doi:10.2147/CMAR.S251820

102. Cui Z, Pu T, Zhang Y, Wang J, Zhao Y. Long non-coding RNA LINC00346 contributes to cisplatin resistance in nasopharyngeal carcinoma by repressing miR-342-5p. Open Biol. 2020;10 (5):190286. doi:10.1098/rsob.190286

103. Tang Y, He X. Long non-coding RNAs in nasopharyngeal carcinoma: biological functions and clinical applications. Mol Cell Biochem. 2021;476(9):3537-3550. doi:10.1007/s11010-02104176-4

104. He J, Zhu S, Liang X, et al. LncRNA as a multifunctional regulator in cancer multi-drug resistance. Mol Biol Rep. 2021;48:1-15. doi:10.1007/s11033-021-06603-7 
105. Wang Q, Zhang W, Hao S. LncRNA CCAT1 modulates the sensitivity of paclitaxel in nasopharynx cancers cells via miR-181a/CPEB2 axis. Cell Cycle. 2017;16(8):795-801. doi:10.1080/15384101.2017.1301334

106. Xia H, Hui K. Mechanism of cancer drug resistance and the involvement of noncoding RNAs. CMC. 2014;21 (26):3029-3041. doi:10.2174/0929867321666140414101939

107. Wong FY, Liem N, Xie C, et al. Combination therapy with gossypol reveals synergism against gemcitabine resistance in cancer cells with high BCL-2 expression. PLoS One. 2012;7 (12):e50786. doi:10.1371/journal.pone.0050786

108. Li L, Gu M, You B, et al. Long non-coding RNA ROR promotes proliferation, migration and chemoresistance of nasopharyngeal carcinoma. Cancer Sci. 2016;107(9):1215-1222. doi:10.1111/ cas. 12989

109. Fan H, Shao M, Huang S, et al. MiR-593 mediates curcumin-induced radiosensitization of nasopharyngeal carcinoma cells via MDR1. Oncol Lett. 2016;11(6):3729-3734. doi:10.3892/ol.2016.4438

110. Kang M, Xiao J, Wang J, et al. MiR-24 enhances radiosensitivity in nasopharyngeal carcinoma by targeting SP1. Cancer Med. 2016;5(6):1163-1173. doi:10.1002/cam4.660

111. Tian Y, Tian Y, Tu Y, et al. microRNA-124 inhibits stem-like properties and enhances radiosensitivity in nasopharyngeal carcinoma cells via direct repression of expression of JAMA. $J$ Cell Mol Med. 2020;24(17):9533-9544. doi:10.1111/jcmm.15177

112. Wang S, Pan Y, Zhang R, et al. Hsa-miR-24-3p increases nasopharyngeal carcinoma radiosensitivity by targeting both the $3^{\prime}$ UTR and 5'UTR of Jab1/CSN5. Oncogene. 2016;35 (47):6096-6108. doi:10.1038/onc.2016.147

113. Zhan S, Ni B. hsa-miR-9-5p down-regulates HK2 and confers radiosensitivity to nasopharyngeal carcinoma. Technol Cancer Res Treat. 2021;20:153303382199782. doi:10.1177/1533033821997822

114. Zhu H, Zhu X, Cheng G, Zhou M, Lou W. Downregulation of microRNA-21 enhances radiosensitivity in nasopharyngeal carcinoma. Exp Ther Med. 2015;9(6):2185-2189. doi:10.3892/ etm.2015.2403

115. Tian Y, Yan M, Zheng J, et al. miR-483-5p decreases the radiosensitivity of nasopharyngeal carcinoma cells by targeting DAPK1. Lab Invest. 2019;99(5):602-611. doi:10.1038/s41374-018-0169-6

116. Zhou X, Zheng J, Tang Y, et al. EBV encoded miRNA BART8-3p promotes radioresistance in nasopharyngeal carcinoma by regulating ATM/ATR signaling pathway. Biosci Rep. 2019;39(9): BSR20190415. doi:10.1042/BSR20190415

117. Ma R, Gao P, Yang H, et al. Inhibition of cell proliferation and radioresistance by miR-383-5p through targeting RNA binding protein motif (RBM3) in nasopharyngeal carcinoma. Ann Transl Med. 2021;9(2):123. doi:10.21037/atm-20-6881

118. Wu W, Chen X, Yu S, Wang R, Zhao R, Du C. microRNA-222 promotes tumor growth and confers radioresistance in nasopharyngeal carcinoma by targeting PTEN. Mol Med Rep. 2017. doi:10.3892/mmr.2017.7931

119. Huang W, Liu J, Hu S, et al. miR-181a upregulation promotes radioresistance of nasopharyngeal carcinoma by targeting RKIP. OTT. 2019;12:10873-10884. doi:10.2147/OTT.S228800

120. Qu J-Q, Yi H-M, Ye X, et al. MiRNA-203 reduces nasopharyngeal carcinoma radioresistance by targeting IL8/AKT signaling. Mol Cancer Ther. 2015;14(11):2653-2664. doi:10.1158/15357163.MCT-15-0461
121. Wang Z, Mao J-W, Liu G-Y, et al. MicroRNA-372 enhances radiosensitivity while inhibiting cell invasion and metastasis in nasopharyngeal carcinoma through activating the PBK-dependent p53 signaling pathway. Cancer Med. 2019;8(2):712-728. doi:10.1002/cam4.1924

122. Qu J-Q, Yi H-M, Ye X, et al. MiR-23a sensitizes nasopharyngeal carcinoma to irradiation by targeting IL-8/Stat3 pathway. Oncotarget. 2015;6(29):28341-28356. doi:10.18632 oncotarget.5117

123. Han Q, Li L, Liang H, Li Y, Xie J, Wang Z. Downregulation of lncRNA X inactive specific transcript (XIST) suppresses cell proliferation and enhances radiosensitivity by upregulating mir-29c in nasopharyngeal carcinoma cells. Med Sci Monitor. 2017;23:4798.

124. Yi L, Ouyang L, Wang S, Li S, Yang X. Long noncoding RNA PTPRG-AS1 acts as a microRNA-194-3p sponge to regulate radiosensitivity and metastasis of nasopharyngeal carcinoma cells via PRC1. J Cell Physiol. 2019;234(10):19088-19102. doi: $10.1002 / j c p .28547$

125. Jin C, Yan B, Lu Q, Lin Y, Ma L. The role of MALAT1/miR-1/ slug axis on radioresistance in nasopharyngeal carcinoma. Tumor Biol. 2016;37(3):4025-4033. doi:10.1007/s13277-015-4227-z

126. Bissey P-A, Teng M, Law JH, et al. MiR-34c downregulation leads to SOX4 overexpression and cisplatin resistance in nasopharyngeal carcinoma. BMC Cancer. 2020;20(1):597. doi:10.1186/s12885-020-07081-z

127. Luo X, He X, Liu X, Zhong L, Hu W. miR-96-5p suppresses the progression of nasopharyngeal carcinoma by targeting CDK1. OTT. 2020;13:7467-7477. doi:10.2147/OTT.S248338

128. Wang G, Wang S, Li C. MiR-183 overexpression inhibits tumorigenesis and enhances DDP-induced cytotoxicity by targeting MTA1 in nasopharyngeal carcinoma. Tumour Biol. 2017;39 (6): 101042831770382. doi:10.1177/1010428317703825

129. Peng X, Cao P, He D, et al. MiR-634 sensitizes nasopharyngeal carcinoma cells to paclitaxel and inhibits cell growth both in vitro and in vivo. Int J Clin Exp Pathol. 2014;7(10):6784

130. Peng X, Cao P, Li J, et al. MiR-1204 sensitizes nasopharyngeal carcinoma cells to paclitaxel both in vitro and in vivo. Cancer Biol Ther. 2015;16(2):261-267. doi:10.1080/ 15384047.2014.1001287

131. Zhao Y, Wang P, Wu Q. miR-1278 sensitizes nasopharyngeal carcinoma cells to cisplatin and suppresses autophagy via targeting ATG2B. Mol Cell Probes. 2020;53:101597. doi:10.1016/j. mcp.2020.101597

132. Huang $\mathrm{L}, \mathrm{Hu} \mathrm{C}$, Chao $\mathrm{H}$, et al. miR-29c regulates resistance to paclitaxel in nasopharyngeal cancer by targeting ITGB1. Exp Cell Res. 2019;378(1):1-10. doi:10.1016/j.yexcr.2019.02.012

133. Gao J, Shao Z, Yan M, Fu T, Zhang L, Yan Y. Targeted regulationof STAT3 by miR-29a in mediating Taxol resistance of nasopharyngeal carcinoma cell line CNE-1. CBM. 2018;22 (4):641-648. doi:10.3233/CBM-170964

134. Zhao M, Luo R, Liu Y, et al. Author Correction: miR-3188 regulates nasopharyngeal carcinoma proliferation and chemosensitivity through a FOXO1-modulated positive feedback loop with mTOR-p-PI3K/AKT-c-JUN. Nat Commun. 2021;12(1):2997. doi:10.1038/s41467-021-22959-7

135. Zheng Z-Q, Li Z-X, Guan J-L, et al. Long noncoding RNA TINCR-mediated regulation of acetyl-CoA metabolism promotes nasopharyngeal carcinoma progression and chemoresistance. Cancer Res. 2020;80(23):5174-5188. doi:10.1158/0008-5472. CAN-19-3626 


\section{Publish your work in this journal}

Cancer Management and Research is an international, peer-reviewed open access journal focusing on cancer research and the optimal use of preventative and integrated treatment interventions to achieve improved outcomes, enhanced survival and quality of life for the cancer patient.

The manuscript management system is completely online and includes a very quick and fair peer-review system, which is all easy to use. Visit http://www.dovepress.com/testimonials.php to read real quotes from published authors.

Submit your manuscript here: https://www.dovepress.com/cancer-management-and-research-journa 